\title{
The Role of Alpha Particles in the Emission of Plasma Waves Inside Solar Ejecta
}

\author{
S. Dasso ${ }^{1,2}$, F.T. Gratton ${ }^{1}$, and C.J. Farrugia ${ }^{3}$ \\ ${ }^{1}$ Instituto de Física del Plasma, CONICET - FCEyN/UBA, \\ Ciudad Universitaria, Pab.1, 1428 Buenos Aires, Argentina \\ ${ }^{2}$ Instituto de Ciencias, Universidad Nacional de General Sarmiento, \\ Campus Universitario, José M. Gutiérrez entre José L. Suárez y Verdi, \\ 1613 Los Polvorines, Prov. de Buenos Aires, Argentina \\ ${ }^{3}$ Institute for the Study of Earth, Oceans, and Space, \\ University of New Hampshire, Durham, NH 03824, USA
}

Received on 26 December, 2001

\begin{abstract}
The enhancement of the resonant instability of right-hand polarized electromagnetic ion cyclotron waves by alpha particles for physical parameters corresponding to coronal mass ejections is studied. We focus on the effects of alpha thermal anisotropy and relative $\mathrm{He}^{++} / \mathrm{H}^{+}$abundance on growth and absorption rates. The first parameter governs directly wave emission, while the second modifies also the wave speed and indirectly enhances the wave excitation.
\end{abstract}

\section{Introduction}

Solar ejecta (also coronal mass ejections, CMEs) are a heterogeneous set of objects with the following plasma properties: (i) low proton plasma beta (see [1] and references therein), (ii) bidirectional streaming (along field lines) of suprathermal electron heat fluxes (few hundreds of $\mathrm{eV})[2]$ and energetic protons $(\sim 1 \mathrm{MeV})[3]$, (iii) a variable $n_{\alpha} / n_{p}$ relative proton-to-alpha particle density which may be much higher than the average solar wind value of $\sim 4 \%[4,5]$, and (iv) a thermal anisotropy $\left(A=T_{\|} / T_{\perp}-1\right)$ which is often negative i.e., $T_{\|}>T_{\perp}$, for both protons and electrons (see Richardson et al., 1997[6] and references therein). The last two properties may hold only in parts of the ejecta. Although physical parameters of CMEs evolve as they propagate away from the sun, they change little on a timescale of several hours. The excitation of right-hand polarized electromagnetic ion cyclotron waves (EICWs) depends sensitively on a number of parameters: the proton beta $\left(\beta_{p}=8 \pi n T_{p} / B^{2}\right)$, the alpha-proton temperature ratio $\left(T_{\alpha} / T_{p}\right)$, the alpha-proton density ratio $\left(\eta_{\alpha}=n_{\alpha} / n_{p}\right)$, the thermal anisotropy of each specie $\left(A_{p}=T_{\|, p} / T_{\perp, p}-1\right.$, for protons, with similar definitions for alpha particles and electrons), and the electron-proton temperature ratio $\left(T_{e} / T_{p}\right)$.

The possibility of right-hand polarized wave activity in CMEs due to the negative proton anisotropy was studied in Farrugia et al., 1998 [7], Gratton et al., 1998
[8], Dasso et al., 1999 [9], Dasso et al., 2000 [10], and Dasso et al., 2001 [11]. Here, we focus on the influence of $A_{\alpha}$ and $\eta_{\alpha}$ on the growth (and absorption) rates of the waves. The former is a little known parameter not yet routinely measured in CMEs. However, on the basis of magnetic moment conservation in an expanding magnetic field, we expect that all particle species will develop a negative thermal anisotropy. Thus, it seems plausible that $A_{\alpha}$ will have the same sign of $A_{p}$, i.e., negative in most solar ejecta. The excitation of right-hand polarized EICWs is due to anomalous Cherenkov emission, and relies on a small number of ions (in the Maxwellian tail) that resonate with the wave[12]. As we shall show, there are frequency ranges where emission overcomes absorption for negative anisotropy. Thus, a negative $A_{\alpha}$, and $\eta_{\alpha}$, contribute directly to the growth rate. However, the effect of $\eta_{\alpha}$ is mostly indirect: adding $\alpha$ increases the inertia of the plasma and thereby reduces the phase velocity of the wave. Thus, more ions (protons and alphas) are brought into resonance. This leads to a considerable enhancement of EICW activity in helium-rich plasmas with respect to low $\eta_{\alpha}$ configurations.

\section{Dispersion Relation}

The instability of right-hand polarized EICWs propagating parallel to the magnetic field is described by the kinetic dispersion relation (see, for example [13]), 


$$
k^{2} c^{2}=\omega^{2}+\sum_{s} \omega_{p s}^{2}\left[A+\frac{(A+1) \omega+A \Omega}{k w_{\|}} Z\left(\frac{\omega+\Omega}{k w_{\|}}\right)\right]_{s},
$$

(summed over species indexed by $s=$ electrons, protons, and alphas; angular frequency $\omega\left[\omega=\omega_{r}+i \gamma\right]$; wavenumber parallel to the ambient field $k$; cyclotron frequency $\Omega$; plasma Zeta function $Z$ ). In (1), all species are modelled by biMaxwellian distribution functions. Here, $\omega_{p s}$ is the plasma frequency; $w_{\|}=$ $\sqrt{2 T_{\|} / m}$, is the thermal speed (temperature in energy units and $m$ is the mass of the particle); and $c$ is the speed of light. Wave quantities vary as $\exp [i(k z-\omega t)]$, where the $z$ axis is aligned with the magnetic field.

In the following, frequencies and growth rates are normalized to the proton cyclotron frequency $x=\omega / \Omega$, where $\Omega=e B_{0} / m_{p} c$ ( $B_{0}$ is the background magnetic field intensity); and the normalized wavenumber $y=k v_{a} / \Omega$, where $v_{a}=B_{0} / \sqrt{4 \pi n_{p} m_{p}}$ is the proton Alfvén velocity. For a plasma composed of protons, alphas, and electrons, Eq. (1) can be written as

$$
\begin{aligned}
y^{2}=A_{p} & +\frac{A_{p}(x+1)+x}{y \beta_{\|, p}^{1 / 2}} Z\left(\frac{x+1}{y \beta_{\|, p}^{1 / 2}}\right)+\eta_{\alpha} A_{\alpha}+\eta_{\alpha} \frac{A_{\alpha}(x+1 / 2)+x}{y \beta_{\|, \alpha}^{1 / 2}} Z\left(\frac{x+1 / 2}{y \beta_{\|, \alpha}^{1 / 2}}\right) \\
& +A_{e} \eta_{e} \mu^{-1}+\eta_{e}^{3 / 2} \mu^{-1 / 2} \frac{A_{e}\left(x-\mu^{-1}\right)+x}{y \beta_{\|, e}^{1 / 2}} Z\left(\frac{x-\mu^{-1}}{y \beta_{\|, e}^{1 / 2}} \eta_{e}^{1 / 2} \mu^{1 / 2}\right) .
\end{aligned}
$$

The following parameters $\beta_{\|, p}=8 \pi n_{p} T_{\|, p} / B_{0}^{2}$, $\beta_{\|, \alpha}=\left(8 \pi n_{p} T_{\|, \alpha} / B_{0}^{2}\right)\left(m_{p} / m_{\alpha}\right), \quad$ and $\beta_{\|, e}=$ $8 \pi n_{e} T_{\|, e} / B_{0}^{2}, \mu=m_{e} / m_{p}$, and $\eta_{e}=n_{e} / n_{p}$ have been introduced. We solve the dispersion relation numerically using an inhouse code for the Zeta function. Parameters are obtained from statistical surveys $[2,6]$ and other case studies. From the roots of the dispersion relation, we obtain the complex dimensionless frequency $x=x_{r}+i g$, as a function of the wavenumber $y$.

\section{Results}

The parameter $n_{\alpha} / n_{p}$ in CMEs is highly variable[2, 6], with $n_{\alpha} / n_{p}$ ranging from very small to values as high as $\sim 15-20 \%[5]$. We consider variations of $A_{\alpha}$ around moderate $A_{p}$ values, chosen here in the range $T_{\|} / T_{\perp}=2-3$. Results of alpha particle effects on the growth and absorption rates of the EICW instability are presented in figures 1-4. Fig. 1 shows from top to bottom the absorption and emission ranges, and the phase speed, all shown as a function of $\eta_{\alpha}$, which parametrizes the various curves. We show results for $\eta_{\alpha}=0, \eta_{\alpha}=0.04, \eta_{\alpha}=0.08$, and $\eta_{\alpha}=0.15$. The electrons are isotropic, $A_{e}=0$. The other parameters are $A_{p}=A_{\alpha}=-0.67, \beta_{p}=0.4$, and $T_{\alpha} / T_{p}=4$. An in- crease in $\eta_{\alpha}$ increases growth rates and shifts the active range to lower frequency. When $\eta_{\alpha}=0.15$, the maximum value of the growth rate is $\gamma / \Omega=8 \times 10^{-4}$ which occurs for $\omega / \Omega=0.56$. This corresponds to an e-folding time $(\tau)$ of 15 minutes for a $15 n T$ magnetic field. Since typical CMEs evolution times are many hours, this $\tau$ value is sufficiently short to allow the instability to develop.

When the absolute value of the alpha particle thermal anisotropy is increased with respect to the case $A_{\alpha}=A_{p}$, an enhacement of growth rates and a broadening of the emission range ensues. In fact, an increment of the parallel temperature of the alpha particles, keeping the average temperature $\left(T_{\alpha} / T_{p}=4\right)$ constant, increases $\beta_{\|, \alpha}$, which in turn augments the instability. This effect can be observed in the Fig. 2, where we vary $A_{\alpha}(=-0.5,-0.58,-0.67,-0.75)$ while keeping other parameters fixed at $A_{p}=-0.67, \beta_{p}=0.4$, $T_{\alpha} / T_{p}=4, \eta_{\alpha}=0.15$. Again $A_{e}=0$. For $A_{\alpha}=-0.75$, the maximum growth rate is $g=2 \times 10^{-3}$, and the unstable $x_{r}$ interval extends from $\sim 0.2$ to $\sim 1.8$. Conversely, when $A_{\alpha} / A_{p}$ is smaller than 1 , the active range is reduced and the low-frequency cut-off of the absorption is shifted to lower frequencies. For example, when $A_{\alpha} / A_{p}$ decreases by $\sim 14 \%$, from $A_{\alpha} / A_{p}=1$ to $A_{\alpha} / A_{p}=0.86$, the beginning of the absorption range is 
shifted to $x_{r} \sim 0.9$. At $x_{r}=1$, we find instability with a growth rate $g=3.5 \times 10^{-4}$ when $A_{\alpha}=A_{p}=-0.67$. When $A_{\alpha}=-0.58$, at the same frequency we find absorption instead, with damping rate $-g=5 \times 10^{-5}$.

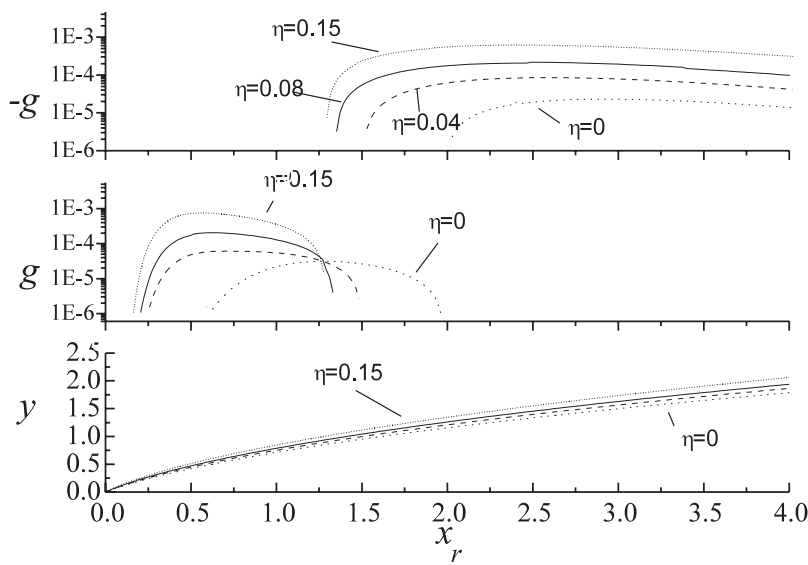

Figure 1. Dimensionless damping rate $-g$, growth rate $g$ and wavenumber $y$ versus dimensionless frequency $x_{r}$ for right-hand polarized EICWs. Dependence on $\eta_{\alpha}$ (with $A_{p}=A_{\alpha}=-0.67, \beta_{p}=0.4, T_{\alpha} / T_{p}=4$, and $A_{e}=0$ constants).
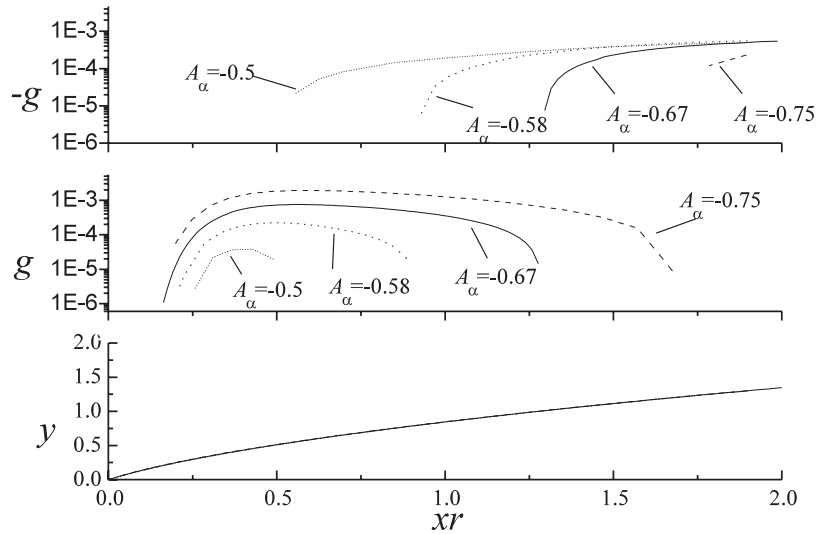

Figure 2. Same as Fig. 1 but showing on $A_{\alpha}$ (with $A_{p}=-0.67, \beta_{p}=0.4, T_{\alpha} / T_{p}=4, \eta_{\alpha}=0.15, T_{e}=T_{p}$, and $A_{e}=0$ constants)

We include the contribution of anisotropic electrons in figures 3 and 4 . Fig. 3 shows a case in which the instability is eliminated (and we obtain absorption instead) when $A_{\alpha}$ goes from $A_{\alpha}=-0.5\left(=A_{p}\right)$ to $A_{\alpha}=-0.4$ keeping $A_{p}=-0.5, \beta_{p}=0.4, T_{\alpha} / T_{p}=4$, $\eta_{\alpha}=0.08, T_{e}=T_{p}$, and $A_{e}=-0.5$ constant.
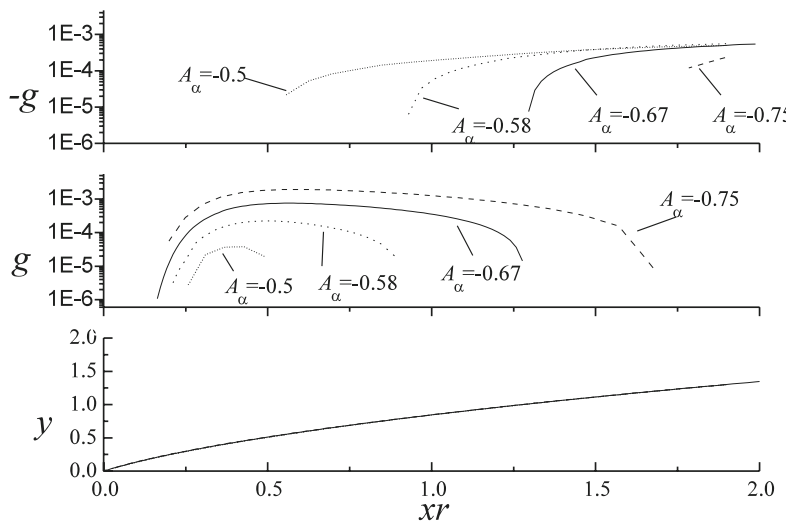

Figure 3. Same as Figure 2, with $A_{p}=-0.5, \beta_{p}=0.4$, $T_{\alpha} / T_{p}=4, \eta_{\alpha}=0.08, T_{e}=T_{p}, A_{e}=A_{p}$ constants.

Somewhat larger absolute values of anisotropies are considered in Fig. 4. Here, we choose $A_{\alpha}=$ $-0.4,-0.5,-0.67,-0.75$, for a configuration with $A_{p}=$ $-0.67, \beta_{p}=0.4, T_{\alpha} / T_{p}=4, \eta_{\alpha}=0.08, T_{e}=T_{p}$, and $A_{e}=-0.67$. A gap in the unstable range (second panel) is formed when $A_{\alpha}=-0.4$. In this gap an absorption band appears.
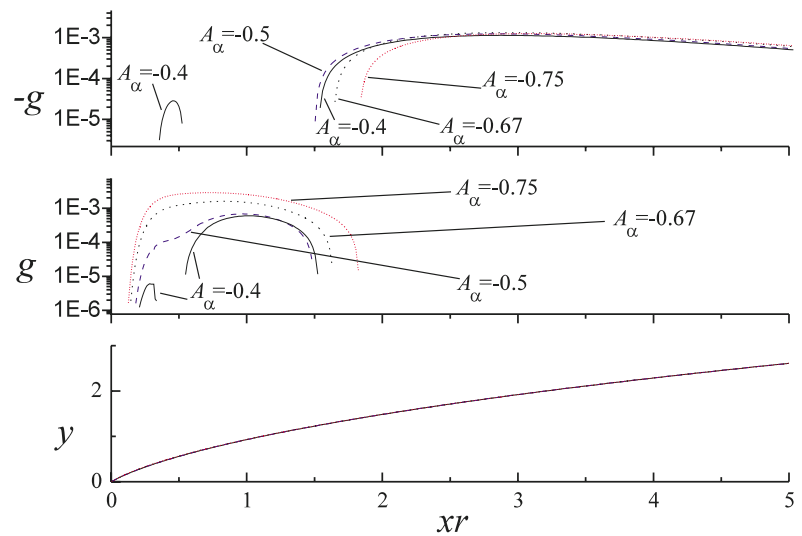

Figure 4. Same as Figure 2, with $A_{p}=-0.67, \beta_{p}=0.4$, $T_{\alpha} / T_{p}=4, \eta_{\alpha}=0.08, T_{e}=T_{p}, A_{e}=A_{p}$ constants.

\section{Conclusions}

Summarizing, we examined the effect of variations in thermal anisotropy and abundance of alpha particles on the growth and absorption rates of EICWs in parameter regimes typical of many CMEs. Increasing $n_{\alpha} / n_{p}$ and/or the alpha-to-proton anisotropy ratio produce enhancements of growth and absorption rates. An increment of $n_{\alpha} / n_{p}$ also shifts the active range to lower frequencies. When the ratio of alpha-to-proton thermal anisotropy increases, an absorption band may appear between two unstable ranges. To advance studies like the one reported here, it would be desirable to measure the alpha particle thermal anisotropy routinely in CMEs, since as we have shown the right-hand polarized EICW instability is very sensitive to this parameter. 
The alpha abundance also influences the growth rate by its effect on the phase velocity of the waves. These properties may help to explain the spectrum of low frequency fluctuations seen in many CMEs by WIND at 1 AU [14].

\section{Acknowledgments}

Work partially supported by Argentinian grants UBACyT (TX032/98 and X059/2001) and CONICET PIP 4536/96, and USA NASA grant NAG5-2834 and living with a star grant NAG5-10883.

\section{References}

[1] J.T. Gosling, in Coronal Mass Ejections, edited by N. Crooker, J.A. Joselyn, and J. Feynman, 9, (AGU, Washington, 1997).

[2] J.T. Gosling, D.N. Baker, S.J. Bame, W.C. Feldman, and R.D. Zwickl, J. Geophys. Res., 92, 8519 (1987).

[3] R.G. Mardsen, T.R. Sanderson, C. Tranquille, K.P. Wenzel, and E.J. Smith, J. Geophys. Res., 92, 11009 (1987).

[4] G. Borrini, J.T. Gosling, S.J. Bame, and W.C. Feldman, J. Geophys. Res., 87, 7370 (1982).

[5] A.B. Galvin, in Coronal Mass Ejections, edited by N. Crooker, J.A. Joselyn, and J. Feynman, 253 (AGU, Washington, 1997).
[6] I.G. Richardson, C.J. Farrugia, and H.V.Cane, J. Geophys. Res., 102, 4691 (1997).

[7] C.J. Farrugia, F.T. Gratton, G. Gnavi, and K.W. Ogilvie, J. Geophys. Res., 103, 6543 (1998).

[8] F.T. Gratton, S. Dasso, and C.J. Farrugia, in Proc. of 1998 Int. Congress on Plasma Physics $8325^{\text {th }}$ European Physical Society Conf on Controlled Fusion and Plasma Physics, Praha, edited by P. Pavlo, ECA, European Physical Society, 22C, 1122 (1998).

[9] S. Dasso, F.T. Gratton, and C. J. Farrugia, in Solar Wind Nine, edited by S. Habbal et al., AIP Conf. Proc., 471, 669-672 (1999).

[10] S. Dasso, F.T. Gratton, C.J. Farrugia, and G. Gnavi, in The Solar Wind-Magnetosphere System 3, 71-80, edited by H. K. Biernat, C. J. Farrugia, and D. F. Vogl, Austrian Academy of Science Press, Vienna (2000).

[11] S. Dasso, C.J. Farrugia, F.T. Gratton, R.P. Lepping, K.W. Ogilvie, and R.J. Fitzenreiter, Adv. Space Res., 28, 5, 747-752 (2001).

[12] C.F. Kennel and F.L. Scarf, J. Geophys. Res., 73, 6149 (1968).

[13] T.H. Stix, Waves in Plasmas (AIM, New York, 1992).

[14] L. Janoo, C.J. Farrugia, R.B. Torbert, J. M. Quinn, A. Szabo, R. P. Lepping, K. W. Ogilvie, R. P. Lin, D. Larson, J. D. Scudder, V. A. Osherovich, and J. T. Steinberg, J. Geophys. Res., 103, 17249 (1998). 